
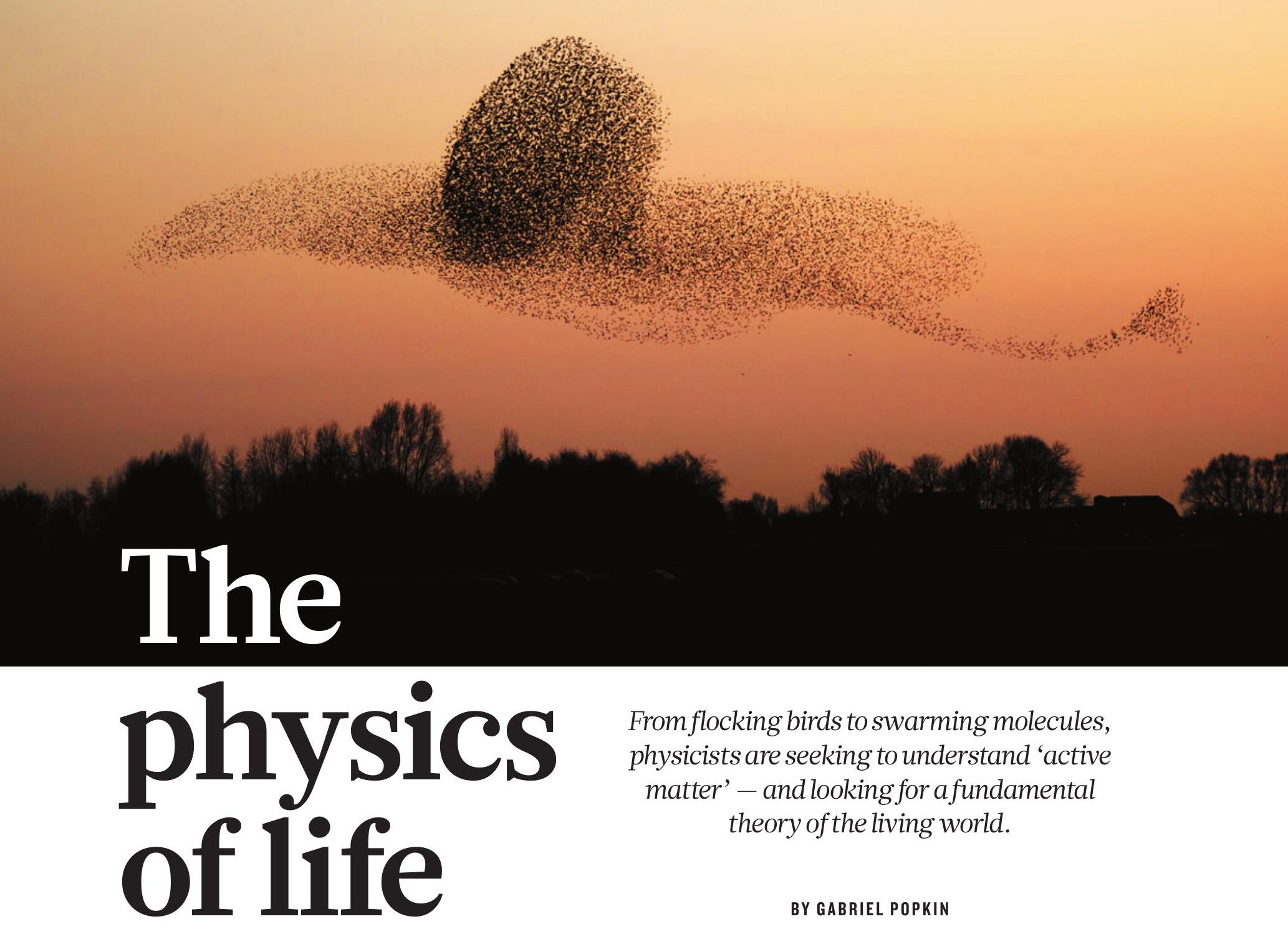

From flocking birds to swarming molecules, physicists are seeking to understand 'active matter' - and looking for a fundamental theory of the living world.

BY GABRIEL POPKIN

$\mathrm{F}$ irst, Zvonimir Dogic and his students took microtubules - threadlike proteins that make up part of the cell's internal 'cytoskeleton' - and mixed them with kinesins, motor proteins that travel along these threads like trains on a track. Then the researchers suspended droplets of this cocktail in oil and supplied it with the molecular fuel known as adenosine triphosphate (ATP).

To the team's surprise and delight, the molecules organized themselves into large-scale patterns that swirled on each droplet's surface. Bundles of microtubules linked by the proteins moved together "like a person crowd-surfing at a concert", says Dogic, a physicist at Brandeis University in Waltham, Massachusetts.

With these experiments, published ${ }^{1}$ in 2012 , Dogic's team created a new kind of liquid crystal. Unlike the molecules in standard liquidcrystal displays, which passively form patterns in response to electric fields, Dogic's components were active. They propelled themselves, taking energy from their environment - in this case, from ATP. And they formed patterns spontaneously, thanks to the collective behaviour of thousands of units moving independently.

These are the hallmarks of systems that physicists call active matter, which have become a major subject of research in the past few years. Examples abound in the natural world among them the leaderless but coherent flocking of birds and the flowing, structure-forming cytoskeletons of cells. They are increasingly being made in the laboratory: investigators have synthesized active matter using both biological building blocks such as microtubules, and synthetic components including micrometrescale, light-sensitive plastic 'swimmers' that form structures when someone turns on a lamp. Production of peer-reviewed papers with 'active matter' in the title or abstract has increased from less than 10 per year a decade ago to almost 70 last year, and several international workshops have been held on the topic in the past year.

\section{THE SECRET OF LIFE}

Researchers hope that this work will lead them to a complete, quantitative theory of active matter. Such a theory would build on physicists' century-old theory of statistical mechanics, which explains how the motion of atoms and molecules gives rise to everyday phenomena such as heat, temperature and pressure. But it could go much further, providing a mathematical framework for stillmysterious biological processes such as how cells move things around, how they create and maintain their shapes and how they divide. "We want a theory of the mechanics and statistics of living matter with a status comparable to what's already been done for collections of dead particles," says Sriram Ramaswamy, a physicist and director of the Tata Institute of Fundamental Research's Centre for Interdisciplinary Sciences in Hyderabad, India.

It could be a while before that want is satisfied, however. Experimentalists are only beginning to gain control of active materials in the lab. Even the most enthusiastic proponents of this research admit that no one has yet produced a theory of active matter that describes the behaviour of everything from cell parts to birds. And if such a theory did exist, it's far from certain that mainstream biologists would see value in it. For biologists, the idea that living matter is active "would be just so obvious as to not really contain very much information", says Jonathon Howard, a molecular biophysicist at Yale University in New Haven, Connecticut.

But that has not kept proponents from imagining applications such as self-assembling 
Flocking birds can synchronize to make patterns.

artificial tissue, selfpumping microfluidic devices and new bioinspired materials although researchers admit that such ideas are still far from being realized. "I think it's too early for the field to have an application, because we're still kind of astonished at what can happen," says Andreas Bausch, a physicist at the Technical University of Munich in Germany - "but I do think the field needs somebody doing it.”

\section{ALL TOGETHER NOW}

All known life forms are based on self-propelled entities uniting to create large-scale structures and movements. If this didn't happen, organisms would be limited to using much slower, passive processes such as diffusion to move DNA and proteins around inside cells or tissues, and many of life's complex structures and functions might never have evolved. Biologists and physicists have speculated for decades about the general principles of living matter, but research on cellular processes has focused on identifying the dizzying array of molecules involved, rather than on working out the principles by which they self-organize. As a result, what is now known as active-matter research did not really get under way until the mid-1990s.

One of the most influential early experiments was conducted by the team of Stanislas Leibler, a biophysicist who was then at Princeton University in New Jersey and is now at the Rockefeller University in New York. The group was among the first to show that complex, life-like structures could self-assemble from microtubules and a few proteins supplied with $\mathrm{ATP}^{2}$. Around the same time, an influential model of active matter was being developed by Tamás Vicsek, a theoretical biophysicist at Eötvös Loránd University in Budapest. In the early 1990s, Vicsek was trying to account for the collective motions of bird flocks, bacterial colonies and cytoskeleton components when he realized that no existing theory would work. "It's not like equilibrium statistical mechanics, where you take a book and you find what to do," says physicist JeanFrançois Joanny of the Curie Institute in Paris.

Instead, Vicsek found a starting point in a model of magnetic materials developed in 1928 by German physicist Werner Heisenberg. Heisenberg imagined each atom as a freely rotating bar magnet, and found that largescale magnetism emerges when interactions between these atomic magnets cause the majority of them to align. To explain active matter, Vicsek replaced the tiny magnets with moving 'arrows' symbolizing particles with velocities that aligned with the average velocity of their neighbours - albeit with a certain amount of random error. That led to what is now known as Vicsek's flocking model ${ }^{3}$. His simulations showed that when enough arrows were packed into a small enough space, they began to move in patterns that closely resembled the familiar movements of bird flocks and fish schools (see 'Smart swarm').

"I got excited," recalls Vicsek, whose 1995 paper $^{3}$ on the model has received more than 3,500 citations. "I was walking up and down the corridor and told people I had designed the moving version of the Heisenberg model."

One physicist attracted to this idea was John Toner, who heard Vicsek give a talk on it in 1994. Toner, now at the University of Oregon in Eugene, saw that Vicsek's swarming arrows could be modelled as a continuous fluid. He took the standard equations for hydrodynamics, which describe fluid flow in everything from tea kettles to oceans, and modified them to account for how individual particles use energy ${ }^{4}$. Toner's fluid model and Vicsek's discrete-particle model gave essentially the same predictions for a wide range of phenomena, and launched a cottage industry of active-matter simulations.

There was only one problem. Whereas the number of simulations was skyrocketing, says physicist Denis Bartolo of the École Normale Supérieure in Lyons, France, "the number of quantitative experiments was constant and very close to zero". Practical work was challenging: no one could hope to do controlled experiments with 10,000 real birds or fish. And at the microscopic scale, few scientists were familiar with both the necessary theoretical work - being published mainly in physics journals - and the biological lab techniques needed to purify cellular components.

\section{PRACTICAL MAGIC}

Only in the late 2000s did the theoretical and experimental pieces begin coming together. Bausch led one of the first precise, quantitative experiments. He and his colleagues mixed actin, a filament that forms most of the cytoskeleton of complex cells, with myosin, a molecular motor that 'walks' on actin and makes muscles contract. The researchers added myosin's natural fuel, ATP, then put the mixture on a microscope slide and watched. "We didn't do anything; we just added the stuff," Bausch says. At low concentrations, the actin filaments swam around without recognizable order. But at higher densities, they formed pulsating clusters, swirls and bands. Bausch and his colleagues immediately recognized and quantified phase transitions of the kind that Vicsek and others had predicted. Their 2010 paper $^{5}$ helped to ignite the experimental active-matter field.

Among the studies that followed were Dogic's 2012 microtubule experiments ${ }^{1}$, which used another walking protein, kinesin. The resulting patterns were much more complex and dynamic than the ones Bausch saw: the flowing microtubules looked like fingerprint whorls in motion. Dogic and his team also noticed that the orderly alignment of this flow would occasionally break down and produce 'defects': discontinuities in the pattern that resemble converging longitude lines at the North and South poles. These defects were dynamic, moving around like self-propelled particles.
No theory at the time could account for this behaviour. But in 2014, Dogic teamed up with Bausch and physicist Cristina Marchetti of Syracuse University in New York to describe the behaviour of active liquid crystals swirling on spherical vesicles in terms of the movement of defects rather than of individual crystal elements ${ }^{6}$. Furthermore, the group found that it could tune the defects' motion by adjusting the vesicle's diameter and surface tension, suggesting a possible way to control an active crystal.

Dogic and his students are now trying to do just that. By studying the spontaneous flows of microtubules and proteins confined in small, doughnut-shaped containers, they hope to lay the groundwork for a self-pumping fluid that could move molecules around in microfluidic devices similar to those that are becoming increasingly common in experimental biology, medicine and industry. Active matter "changes our ideas of what materials can do", says Dogic.

But any industrial application will have to overcome at least one major roadblock. The biological materials currently used in active-matter experiments are expensive and time-consuming to purify - Dogic's microtubules come from cow brains, and Bausch uses actin cC We're from rabbit muscle - and they last still kind of only a short time in the lab. Until a astonished cheap, robust, offthe-shelf source at what can happen." of active-matter materials can be found, commercial use is unlikely, says Bausch.

Advances in synthetic active materials may show the way forward. In 2013, New York University physicist Paul Chaikin and his colleagues described making particles of haematite, an iron oxide mineral, inside a spherical polymer ${ }^{7}$. When the scientists placed these 'swimmers' in a solution of hydrogen peroxide and exposed them to blue light, a chemical reaction caused the particles to move around spontaneously, clumping and unclumping like groups of people at a cocktail party.

In 2013, Bartolo and his colleagues reported large-scale flows using even simpler plastic spheres in a conducting fluid ${ }^{8}$. When the researchers turned on an electric field, the spheres began rotating in random directions. At high enough densities, interactions between nearby spheres caused them to spontaneously roll, flock-like, in the same direction.

Such lab-made materials remain primitive, however, compared with those produced in cells by 4 billion years of evolution. Dogic notes that the kinesins he uses are much more efficient than any human-made motors at converting energy to motion. And Bartolo is also quick to discourage talk of short-term pay-offs. "I'm not targeting a specific application," he 


\section{Smart swarms}

A simple model of interactions among self-propelled particles can realistically simulate the movement of flocks of birds, schools of fish, self-assembling proteins in the cell and many other forms of active matter.

\section{Low density: randomness} When individuals have few neighbours to compare themselves to, they mil about with no obvious pattern.

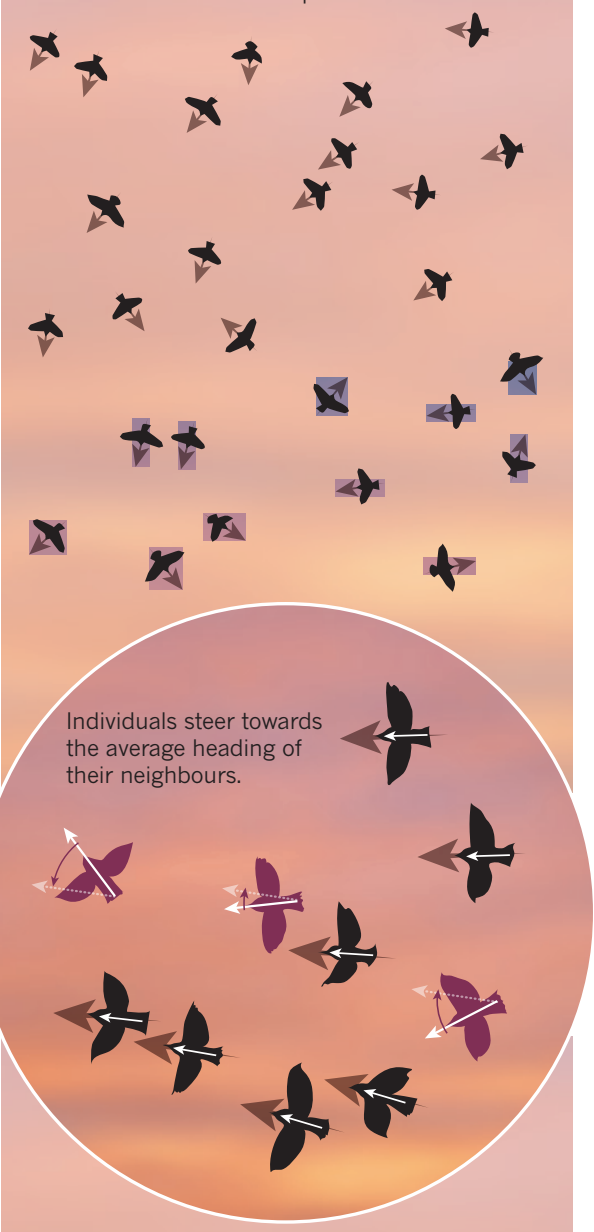

Higher density: flocking As the density increases, the gro motion becomes synchronized.

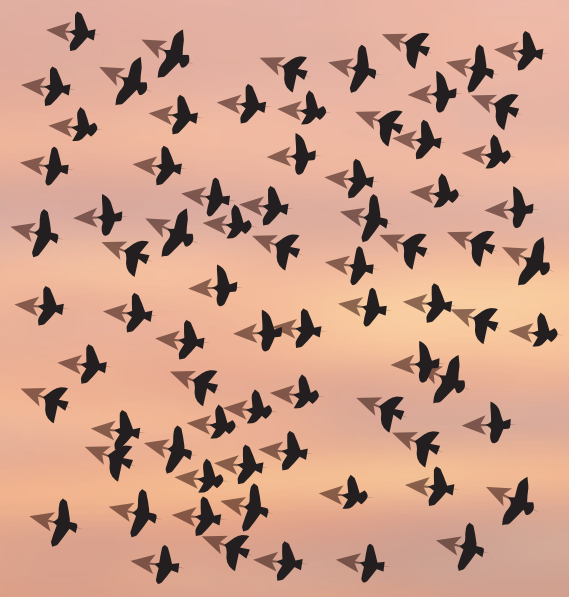

says of his rotating plastic spheres.

Possible applications aside, active matter excites scientists because it so closely resembles the most complex self-organizing systems known: living organisms. In 2011, Dogic and his colleagues reported ${ }^{9}$ that microtubule bundles anchored at one end to air bubbles on a microscope slide beat in synchronized, wave-like patterns eerily reminiscent of the hair-like cilia and flagella that protrude from the surfaces of some cells. And in his 2012 paper ${ }^{1}$, he noted a striking similarity between his microtubule flows and cytoplasmic streaming, a process in which cytoskeletal filaments team up to whisk a cell's contents around like "a giant washing machine", he says.

The resemblance between lab-prepared active matter and living things can be uncanny, agrees Jennifer Ross, a physicist at the University of Massachusetts Amherst. At talks, she has shown videos of spherical microtubulekinesin systems and asked audience members whether they think they are seeing a real cell. "Whenever I present these to cell biologists in particular, they are always fooled," she says.

But something can look and act like a living organism without actually following the same rules, cautions Howard. He points out that Dogic's group created something that looks and acts very much like a cilium or flagellum with its multitude of proteins - but that may, in fact, work very differently. "There's something in there about the underlying mechanism, but it's extremely abstract," he says.

\section{IS IT ENOUGH?}

To probe whether active-matter theory can reveal biological mechanisms, Daniel Needleman, a biophysicist at Harvard University in Cambridge, Massachusetts, studied the spindle: a microtubule-based structure that controls the separation of chromosomes during cell division. He wanted to test the idea, suggested by earlier theories and experiments, that short-range microtubule-kinesin interactions by themselves were sufficient to yield spindle-like structures. He first used sophisticated microscopes to examine extracts from frog egg cells, quantifying microtubule density, orientation and stresses during spindle formation. "It really was not clear at all until Dan came along that you could measure all these things," says Howard.

Needleman then merged his measurements with models of how active matter self-organizes. In 2014, he and Jan Brugués, a biologist at the Max Planck Institute of Molecular Cell Biology and Genetics in Dresden, Germany, reported that, consistent with theory, the interactions they observed among closely spaced microtubules are enough to produce the spindle and keep it stable ${ }^{10}$. "People have argued that you need more complex processes," says Needleman. "But the fact that one can understand so much of the spindle without invoking any of that shows that it's certainly not necessary."

Others are using ideas from active matter to probe how large numbers of cells organize in processes such as tissue growth, wound healing and the spread of tumours. Theorists including Marchetti, Joanny and Frank Jülicher of the Max Planck Institute for the Physics of Complex Systems in Dresden have modelled tissues ${ }^{11}$ and tumours ${ }^{12}$ as flowing cells that self-organize through short-range cell-to-cell interactions rather than chemical signals. Experimentalists are testing such ideas, for instance, by showing that active-matter theory can help to describe cell organization in a developing fruit-fly wing ${ }^{13}$.

Some biologists hope that such studies will reveal the fundamental principles that govern how cells divide, take shape or move. "It's like Linnaean classification before Darwin came along," says biologist Tony Hyman of the Max Planck Institute of Molecular Cell Biology and Genetics. "We've got all these molecules, just like they had all those species, and we need to put some kind of order, some kind of reason behind it all." Active matter, Hyman thinks, could provide that reason.

But even enthusiasts admit that mainstream biologists may need convincing. "We used to get a lot of papers rejected at the beginning," says Hyman - in part because the manuscripts' heavy use of mathematics made it hard to find reviewers. Even the phrase 'active matter' may hinder communication, adds Howard. "It's kind of a physics-y term."

Still, Howard and Hyman hope that acceptance will be aided by increasing convergence between fields. Among biologists, says Hyman, "I think the new generation coming along will be trained in physics from the beginning."

And that's good, adds Stephan Grill, a biophysicist at the Technical University of Dresden, because progress in active matter calls for scientists who are at the cutting edge of both physics and biology. "The pot of gold is at the interface," he says, "but you have to push both fields to their limits." -

Gabriel Popkin is a freelance writer in Mount Rainier, Maryland.

1. Sanchez, T., Chen, D. T. N., DeCamp, S. J. Heymann, M. \& Dogic, Z. Nature 491, 431-434 (2012).

2. Nédélec, F. J., Surrey, T., Maggs, A. C. \& Leibler, S. Nature 389, 305-308 (1997).

3. Vicsek, T., Czirók, A., Ben-Jacob, E., Cohen, I. \& Shochet, O. Phys. Rev. Lett. 75, 1226-1229 (1995)

4. Toner, J. \& Tu, Y. Phys. Rev. Lett. 75, 4326-4329 (1995).

5. Schaller, V., Weber, C., Semmrich, C., Frey, E. \& Bausch, A. R. Nature 467, 73-77 (2010).

6. Keber, F. C. et al. Science 345, 1135-1139 (2014).

7. Palacci, J., Sacanna, S., Steinberg, A. P., Pine, D. J. \& Chaikin, P. M. Science 339, 936-940 (2013).

8. Bricard, A., Caussin, J.-B., Desreumaux, N., Dauchot, O. \& Bartolo, D. Nature 503, 95-98 (2013).

9. Sanchez, T., Welch, D., Nicastro, D. \& Dogic, Z. Science 333, 456-459 (2011).

10.Brugués, J. \& Needleman, D. Proc. Natl Acad. Sci. USA 111, 18496-18500 (2014).

11.Ranft, J. et al. Proc. Natl Acad. Sci. USA 107 20863-20868 (2010)

12.Basan, M., Risler, T., Joanny, J.-F., Sastre-Garau, X., \& Prost, J. HFSP J. 3, 265-272 (2009).

13.Aigouy, B. et al. Cell 142, 773-786 (2010). 